\title{
THE INFLUENCE OF STRUCTURE OF MOBILE CONSTRUCTION MANIPULATORS ON THEIR KINEMATIC AND DYNAMIC CHARACTERISTICS
}

\author{
Stanisław Michałowski ${ }^{a}$, Andrzej Sobczyk ${ }^{b}$, Witold Trzaska $^{c}$ \\ ${ }^{a}$ Cracow University of Technology,POLAND,pmmichal@cyf-kr.edu.pl \\ ${ }^{\mathrm{b}}$ Cracow University of Technology,POLAND, sobczyk@mech.pk.edu.pl \\ ${ }^{\mathrm{c}}$ Cracow University of Technology,POLAND, wtrzaska@mech.pk.edu.pl
}

\begin{abstract}
For the purpose of studies, a model of a wheeled, construction manipulator was developed and dully analysed. Experience shows that the presence of hydraulic driving systems is the major determinant of large-scale stationary and mobile manipulators' flexibility. In terms of dynamics, the major problem is control of natural vibrations during transient state motion because of machine's stability or during its ride to provide the required precision of position control. The results presented here suggest that while modelling hydraulic drive and steering systems for such machines, it is necessary to develop and analyse more complex mathematical models which would include also the effects of the internal structure of the system on its dynamic behaviour.
\end{abstract}

Keywords: construction manipulators, flexibility, active damping, position control

\section{INTRODUCTION}

Before manipulators can be used in assembly and construction it is necessary to solve certain problems involving the working conditions and the distinctive features of this type of machines. With regards to the specificity of construction jobs, the manipulators can be divided into the following groups: earthmoving, assembly, building erection and construction manipulators. Because of the applied control systems modern manipulators can be roughly divided into those with force feedback, those with direct control and those with remote control and visual observation of the working field or with provided vision systems with programmable duty cycles. That group includes manipulators used in building industry. In terms of kinematic structure these manipulators can be treated as mechanisms made of several stiff and elastic links connected with sliding or rotating kinematic pairs. From the point of view of topology, a manipulator globally is an open kinematic chain, with locally closed links from the steering and control system. Problems arising in construction and assembly are: design problems involving the strength and elasticity of bearing elements and gripping devices, providing position and force control whilst the working field and loading may not be always predetermined [3], selection of driving systems suitable for working massive, large scale manipulator components; also modelling structural chains, characteristic of the drive systems and measuring circuits. Mobile manipulators used in road building and maintenance as well as bridge inspection and repairs make a special group. They require precise control of working equipment position during their ride, in the conditions of chassis vibrations generated when the machine moves over the rough or uneven terrain. Experience shows that the presence of hydraulic driving systems is the major determinant of largescale stationary and mobile manipulators' flexibility. Such systems are applied there to provide for smooth transmission of considerable forces. In terms of kinematics, are faced with the problem of precise end point positioning within the working field. In terms of dynamics, the major problem is control of natural vibrations during transient state motion because of machine's stability or during its ride to provide the required position control precision.

\section{INFLUENCE OF FLEXIBILITY ON POSITIONING OF LARGE-SCALE MOBILE MANIPULATORS}

In mobile manipulators used in building industry the actuator link is usually installed on the working equipment of the machine, for example the hydraulic shovel. In the working equipment of mobile construction machines the flexibility of liquid links is usually much greater than that of solid links [4]. Hence we can assuming without a risk of major errors that only liquid links (hydraulic cylinders) will be flexible, while the other links will not be deformed. Though the hydraulic drive can be 
implemented through various, often very complex, hydraulic systems, the structure of drive sub-system of a single cylinder can be easily brought down to the simplified diagram presented in Fig 1. For such a sub-system, the equivalent cylinder flexibility can be determined taking into account working fluid compressibility and deformability of elastic hose.

Assuming that: $D$ - piston diameter; $d$ - piston rod diameter; $L_{I}, L_{I I}$ - length of the elastic hose on the piston end and piston rod end, $\beta$ - coefficient of fluid isothermal compressibility; $V_{\text {Imin }}$ - minimal volume of

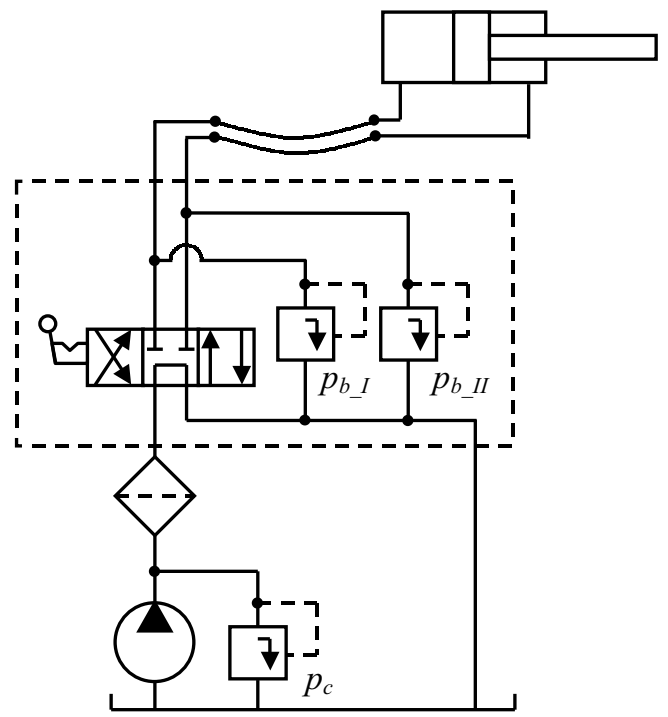

Fig 1. Diagram of typical cylinder drive subsystems.

liquid on the piston end while the cylinder is closed (from the distributor to the piston); $V_{\text {IImin }}$ - minimal volume of liquid on the piston rod end while the cylinder is open (from the distributor to the piston); $d_{p^{-}}$internal diameter of the elastic hose; $\beta_{w^{-}}$average hose extensibility coefficient ; $x$ - piston displacement measured from the cylinder bottom to the piston front $(x=0 . . s), s$ - cylinder stroke, then the cylinder flexibility under compressing loads can be obtained from the formula:

$$
k_{I}=\frac{4 \beta}{\pi D^{2}} x+\frac{16 \beta V_{I_{\min }}+4 \pi \beta_{w} L_{I} d_{p}^{2}}{\pi^{2} D^{4}}
$$

Cylinder flexibility under tensile loading will be:

$$
\begin{aligned}
& k_{I I}=\frac{-4 \beta}{\pi\left(D^{2}-d^{2}\right)} x+ \\
& +\frac{4 \pi \beta_{w} L_{I I} d_{p}^{2}+16 \beta V_{I I_{\min }}+4 \pi \beta s\left(D^{2}-d^{2}\right)}{\pi^{2}\left(D^{2}-d^{2}\right)^{2}}
\end{aligned}
$$

\subsection{Mathematical model}

The computation model for a 6 DOF manipulator is presented in Fig 2.

For small displacements the motion of such a system can be described with a linear differential equation in the for of a matrix:

$$
\boldsymbol{M} \cdot \frac{d^{2}}{d t^{2}} \boldsymbol{q}+\boldsymbol{D} \cdot \frac{d}{d t} \boldsymbol{q}+\boldsymbol{C} \cdot \boldsymbol{q}=\boldsymbol{F}
$$

where: $\boldsymbol{M}$ - inertia matrix, $\boldsymbol{D}$ - damping matrix, $\boldsymbol{C}$ rigidity matrix, $\boldsymbol{q}$ - vector of generalised coordinates

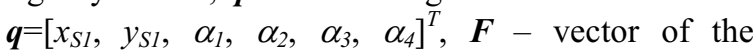
generalised forces.

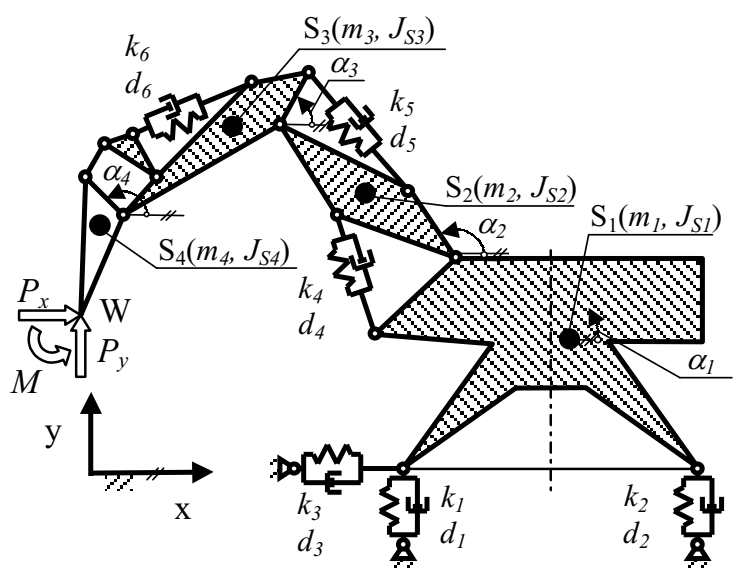

Fig 2. Model of a construction manipulator

Having found the vector of coordinates $\boldsymbol{q}$ from (3) for the given initial conditions and the generalised forces, we can now define the position of the manipulator wrist on the basis of coordinates of any characteristic point $\mathrm{W}\left(x_{W}, y_{W}\right)$ and the wrist orientation angle $\alpha_{4}$.

Dynamic errors are defined as the difference between the values obtained when the system is assumed to be inflexible (*) and those obtained for flexible systems in dynamic conditions $(d)$.

$$
\left[\begin{array}{c}
\Delta x_{W_{d}} \\
\Delta y_{W_{d}} \\
\Delta \alpha_{4_{d}}
\end{array}\right]=\left[\begin{array}{c}
x_{W_{*}} \\
y_{W_{*}} \\
\alpha_{4_{*}}
\end{array}\right]-\left[\begin{array}{c}
x_{W_{d}} \\
y_{W_{d}} \\
\alpha_{4_{d}}
\end{array}\right]
$$

In static conditions, in accordance with Fig. 2, the positioning errors can be obtained from the matrix equation:

$$
\left[\begin{array}{c}
\Delta x_{W_{s}} \\
\Delta y_{W_{s}} \\
\Delta \alpha_{4_{s}}
\end{array}\right]=\boldsymbol{K}_{\boldsymbol{P}} \cdot\left[\begin{array}{c}
P_{x} \\
P_{y} \\
M
\end{array}\right]+\boldsymbol{K}_{\boldsymbol{G}} \cdot\left[\begin{array}{l}
G_{1} \\
G_{2} \\
G_{3} \\
G_{4}
\end{array}\right]
$$


where: $\boldsymbol{K}_{\boldsymbol{P}}-$ flexibility matrix related to the vector of wrist loading due to manipulator operation; $\boldsymbol{K}_{\boldsymbol{G}}-$ flexibility matrix related to forces of gravity, $G_{i}-$ force of gravity for the element $i=1 . .4$.

As manipulator displacement is to be determined from the static balance position of a flexible system, the second component in $\mathrm{Eq}(3)$ expressing weight of system elements should be omitted. Besides, the reference level in Eq (3) should be related not to the inflexible system position, but from the static balance position of a flexible systems.

\subsection{Numerical example}

The analysis was performed for a manipulator installed on a wheeled hydraulic shovel with the bucket capacity $0.4 \quad\left[\mathrm{~m}^{3}\right]$. Shovel structure corresponds to the model presented in Fig 2. Cylinder flexibility was determined for tensile and compressing loads while a typical hydraulic oil having the isothermal coefficient of fluid compressibility $\beta=7.5 \cdot 10^{-10}[1 / \mathrm{Pa}]$ [5] or water with $\beta=4.75 \cdot 10^{-10}[1 / \mathrm{Pa}][6]$, are used as working fluid, the pressure range $0-20[\mathrm{MPa}]$. The average hose extensibility coefficient is taken to be $\beta_{w}=10 \cdot 10^{-10}$ $[1 / \mathrm{Pa}][5]$.

A diagram of boom cylinder flexibility $\left(k_{4}\right)$ in the condition of piston-end and piston rod- end loading for hydraulic oil and water is presented in Fig 3.

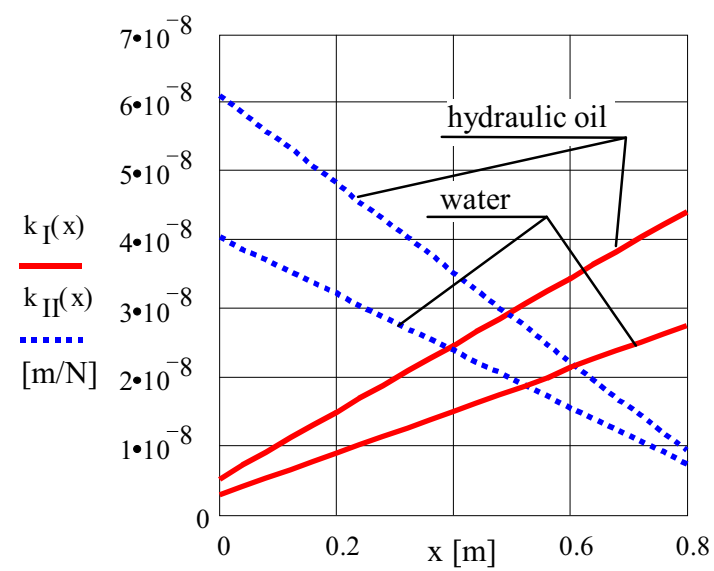

Fig. 3. Boom cylinder flexibility $\left(k_{4}\right)$ for hydraulic oil and water

Several models of the analysed system were developed for computer simulation, using the computer programme Working Model ${ }^{\circledR}$ 2D. These models help to simulate the operations of a wheeled construction manipulator:

- during the ride, hence they have to provide kinematic excitations resulting from rough terrain

- when machine is at the standstill on flexible supports or on wheels.
The cylinder model shown in Fig 4 was developed to take into account the variable flexibility of individual actuators. In this case the actuator 1 acts as a drive cylinder while actuator 2 may be used to introduce friction forces. The separator and the rope allow for limitation of the maximal and minimal cylinder length and provide for an abrupt change of cylinder flexibility in its extreme positions. Actually, the cylinder length is equal to the distance between the joints $\mathrm{A}$ and $\mathrm{B}$. This length impacts on the spring stiffness.

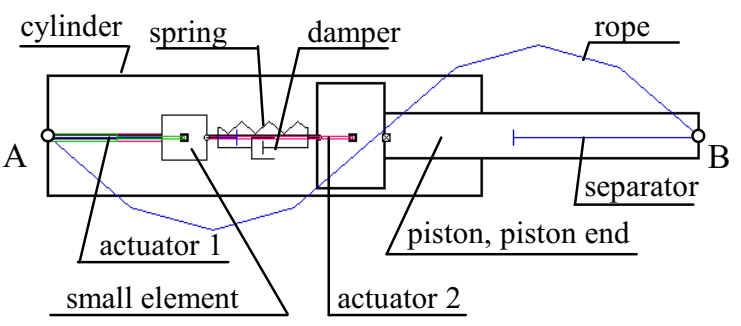

Fig. 4. Model of a hydraulic cylinder in WM

A case of manipulator operation is presented in Fig 5 . It corresponds to the situation when a load falls from the manipulator wrist. The equivalent flexibility of tires is taken to be $k_{1}=k_{2}=k_{3}=3.3310^{-7}[\mathrm{~m} / \mathrm{N}]$. Calculations use mass distributions, masses, mass moments of inertia the same as in the real (hydraulic shovel K-406 manufactured by BUMAR-Waryński). The manipulator wrist is loaded with the vertical force $P_{y}=-10000[\mathrm{~N}]$, which is disappears after $1[\mathrm{~s}]$ The starting point in the analysis is the static balance position. Vertical displacements of the point $\mathrm{W}$ and boom cylinder loading are shown in Fig 5.

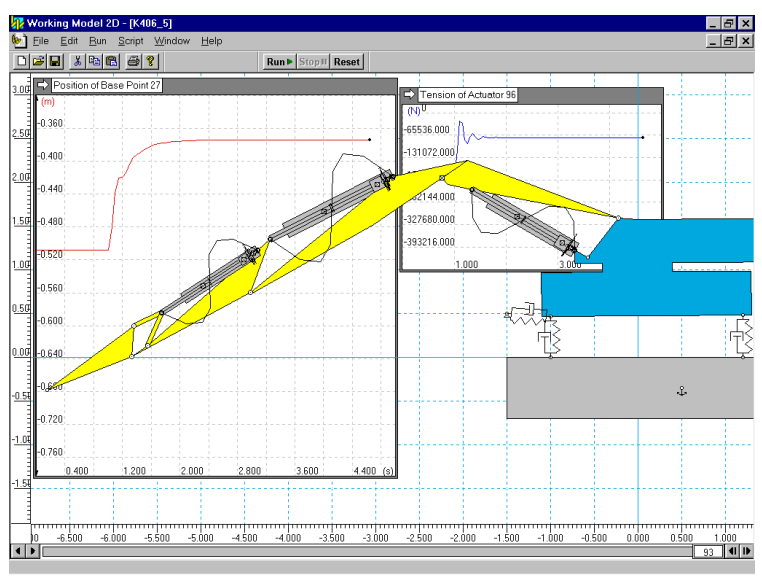

Fig. 5. Computer simulation

Studies reveal that minimisation of positioning errors through application of water as the working fluid has significant effects only when the machine is used on surfaced ground, when it is on inflexible supports. The positioning errors in this case vary in proportion to the changes of bulk modulus of the working fluid. 
Application of water-based hydraulic systems brings significant environmental benefits, which is of primary importance in erection of engineering structures when the environmental requirements are more stringent. It can be expected that in the years to come the environmental standards will be getting still stricter.

\section{POSITION CONTROL OF MOBILE MANIPULATOR END POINT}

One of the objectives of manipulator design is to eliminate vibrations of manipulator end link, which may adversely affect its functional features. Whilst the machine is in motion, the intensity of end link vibrations over the range $0-10 \mathrm{~Hz}$ depends on the terrain features, geometry and mass parameters of the machine frame, properties of the steering system and parameters of the equipment. It follows from the research that these vibrations ought to be reduced

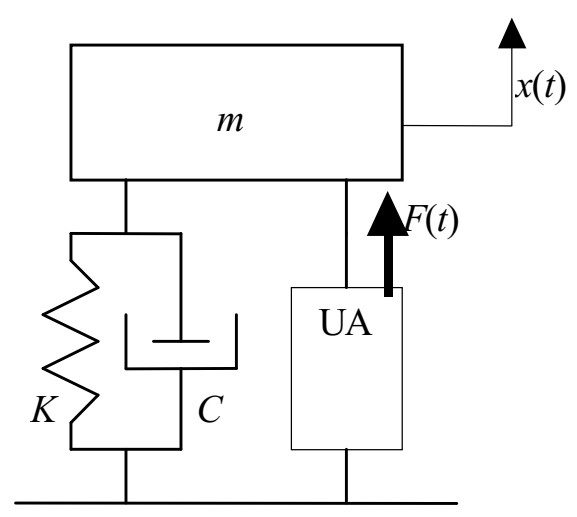

Fig 6. Physical model of the system

through control systems in equipment actuators drives, that is active vibro-control systems. Because of the applied driving systems in mobile manipulators it possible to use electro-hydraulic control systems. In case of manipulators used in road works, operating in rough terrain, it is necessary to eliminate vertical oscillations of the working tools ranging from $0-1.5 \mathrm{~Hz}$, for amplitudes of output link displacement being of the order of $1 \mathrm{~m}$. Vibro-control systems for such small frequencies ought to keep the end link in a precisely controlled position with respect to the co-ordinate system determined by the terrain features. Gyroscopes and laser systems can be often found in these applications. The basic problem that arises here is ensuring that the time constant is so small that the delay in compensating force or displacement generation will not make the whole system unstable. Dynamic characteristics of actuators with liquid links is determined to a large extent by friction forces in joints and cylinders. Manipulator equipment links are large and have complex geometry, so in terms of dynamics they can be modelled as solids throughout the range of low- frequency vibrations. Therefore, still providing the dynamic similarity, we can apply the discrete model taking into account flexibility of drive system cylinders. Thorough examination of various control systems in electro-hydraulic actuators providing position control of the output link is of primary importance because of nonlinearity of actuators' characteristics. Linearisation of the mathematics model may simplify the controller selection process, yet it may also lead to major discrepancies between the theoretical results and those obtained in practical applications.

\subsection{Mathematical model}

The analysis of dynamic phenomena in mobile manipulators is based on mathematical models which offer several degrees of structure simplification. To ensure that the machine should function properly, it is important to investigate how to damp the lowest frequency vibrations, which are accompanied by most significant displacements. That means vibrations of a one-DOF system, assuming that this reduction should involve the co-ordinate related to the piston rod motion of the driving cylinder, being the actuator in an active vibro-control system. Fig 6 presents the most simplified model of the object with the mass $m$, whose natural vibrations are reduced through an active system. $F(t)$ stands for the unknown compensating force generated by the actuator. The remaining parameters of thus simplified system are related to the first frequency of manipulator vibrations and describe how the free vibrations cease. They are denoted by $C$ and $K$, respectively. Absolute displacement of a given equipment link point within the co-ordinate system related to the actuator cylinder is designated as $x(t)$. Differential equations of motion of thus simplified system can be written as:

$$
\frac{d^{2} x}{d t^{2}}+2 \omega_{0} \zeta_{0} \frac{d x}{d t}+\omega_{0}^{2} x=f(t)
$$

where: $2 \omega_{0} \zeta_{0} m=K, \omega_{0}^{2} m=C, m f(t)=F(t)$.

For the object represented by the mathematical model described with (6) we seek the optimal compensating force $F(t)$ to provide the most favourable condition for damping of natural or kinematic- mode excited vibrations, whose frequency is close to the first natural frequency of the manipulator [2]. The force $F(t)$ is generated by an electro-hydraulic actuator, which is an amplifier with a continuous voltage or current input signal, the output signal being the variable flow rate or pressure of the working liquid. For low- frequency vibrations the equation describing the actuator can be rewritten as [1]: 


$$
\begin{aligned}
& \frac{V_{k}}{E_{c} A} \frac{d F(t)}{d t}+\frac{k_{p}}{A} F(t)+A \frac{d x(t)}{d t}= \\
& =k_{z} z(t)\left\{p_{0}-p_{s}-\frac{F(t)}{A} \operatorname{sgn}[z(t)]\right\}^{n}
\end{aligned}
$$

where: $V_{k}, E_{c}$ - volume and elasticity modulus of the working liquid, respectively; $A$ - effective area of the piston; $p_{o^{-}}$constant pressure in the supply line, $p_{s^{-}}$ support pressure in the return line; $k_{p^{-}}$coefficient relating to leaking intensity in the cylinder; $k_{z^{-}}$ coefficient relating to throttling characteristics of the distributor; $z(t)$ - displacement of the distributor spool, $n$ - power exponent depending on the type of low in distributor slots.

One of the major problems involved in selecting the parameters of active vibro-control systems is finding the relation between displacement $z(t)$ and kinematic parameters of the structure whose vibrations are to be damped. A common solution here is providing active control through signals proportional to the linear velocity of the given end link point. Hence, the following formula [4] can be utilised:

$$
z(t)=-K_{v} \frac{d x(t)}{d t}
$$

where: $K_{v}$ - feedback loop gain coefficient.

Taking into account all the previous assumptions and the linear approximation of Eq (7), we can study the effectiveness of damping, assuming the structure will produce harmonic vibrations due to acceleration of translation, being the consequence of manipulator motion over uneven terrain. To determine the minimal amplitude of manipulator vibrations it would be necessary to compute the gain coefficient $K_{v}$ for specified values of remaining parameters characterising the manipulator and actuator.

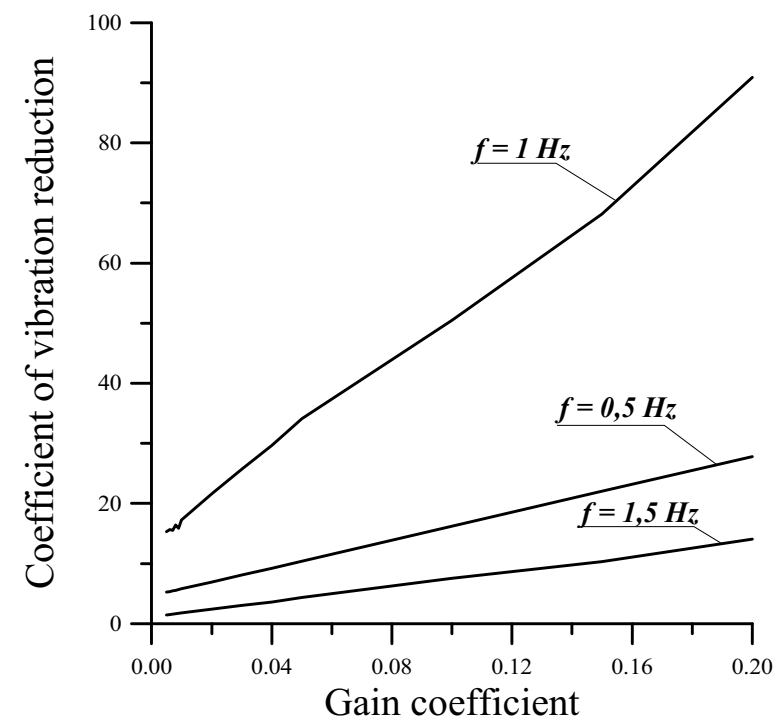

Fig. 7. Relations between vibration reduction coefficient and gain coefficient for different frequencies

\subsection{Results of the analysis}

Selected results of computer simulation using MATLAB $^{\circledR}$ software are presented in Fig 7. They show the changes in characteristics of active vibrocontrol systems. Parameters for the calculations were as follows: reduced mass of the working equipment and end link $m=1000[\mathrm{~kg}]$; natural frequency about 1 $\mathrm{Hz}$; damping coefficient $\zeta_{0}=0.1$. The following parameters of the actuator were considered: $V_{k}=0.04$ $\left[\mathrm{m}^{3}\right] ; E_{c}=300[\mathrm{MPa}], A=0.0113\left[\mathrm{~m}^{2}\right] ; p_{0}=5[\mathrm{MPa}] ;$

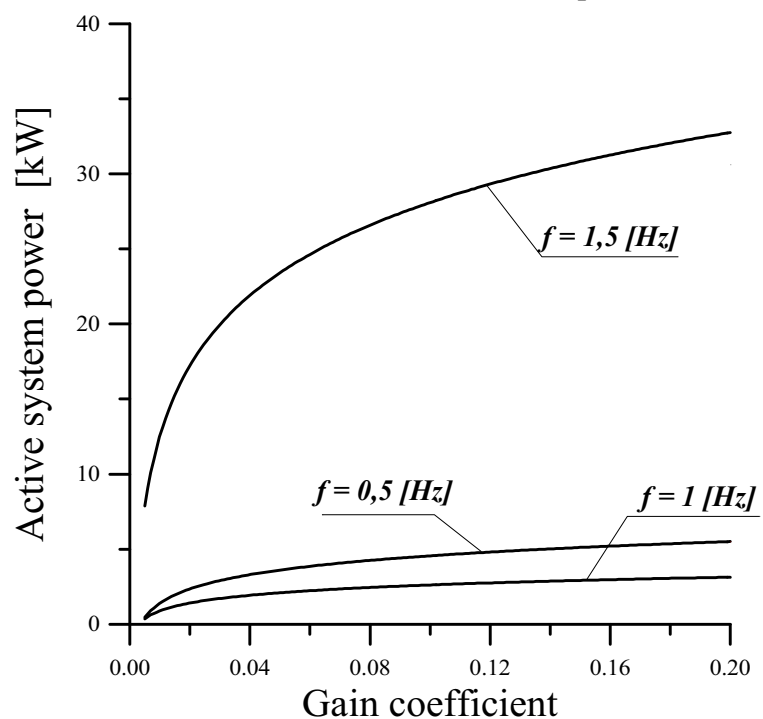

Fig. 8. Relations between power of active system and gain coefficient, for different frequencies

$p_{s}=2[\mathrm{MPa}] ; k_{p}=3.510^{-10}\left[\mathrm{~m}^{4} \mathrm{~s} / \mathrm{kg}\right] ; k_{z}=1,7510^{-4}$ $\left[\mathrm{m}^{3} \mathrm{~s} / \mathrm{kg}\right]$.

The amplitude of system vibrations was kept constant for the whole range of excitation frequencies, with no active vibro-control. The curves in Fig 7 are hardly any different from the linear relation. The active vibro-control system proves to be most effective in damping resonance vibrations. Fig 8 presents the relations between the gain coefficient and the power of the active system, obtained from the maximal flow rate of the working liquid to the cylinder at preset pressure.

\section{CONCLUSIONS}

This analysis of manipulator end link properties may be the basis for evaluating their efficiency, in the light of requirements for manipulator end link position control during its operation and ride. These observation lead to more general conclusions, too. First of all, while modelling such systems we have to take into account the characteristics of connections between the mechanical components, flexibility of structure and driving systems, we must not limit our considerations to mass and moment of inertia. Such 
an approach seems vital particularly now, when we have to design driving systems for large scale construction manipulators or working equipment tools positioning systems in heavy machines.

The results presented here suggest that while modelling hydraulic drive and steering systems for such machines, it is necessary to develop and analyse more complex mathematical models which would include also the effects of the internal structure of the system on its dynamic behaviour. Such problems may be difficult to predict beforehand. When the existing machines assumes manipulator functions, its structure must be considered - otherwise the machine may not function properly. Furthermore, that determines the possibility of utilising the system for given jobs, defined in terms of quality and quantity.

\section{REFERENCES}

[1] Baranov W.N.: Electro-hydraulic Follow-up Control of Vibratory Machines. Mashinostroyenye. Moscow 1988, (in Russian).

[2] Górecki H.: Dynamic Systems Optimisation. PWN Warsaw 1993, (in Polish).

[3] Komainda A., Hiller M.: Motion Control of Redundant Large-scale Manipulators in a Changing Environment - Proc. of 10-th World Congress on the Theory of Machines and Mechanisms, Oulu, Finland, June 20-24,1999, pp. 1033-1038.

[4] Michałowski S.: Active Systems in Construction of Heavy-duty Machines. Cracow University of Technology Publishers, Monograph No 171, 1994, (in Polish).

[5] Stryczek S.: Hydrostatic Transmission Components (v.1), WNT, Warsaw 1995, (in Polish).

[6] Trostmann E.: Water Hydraulics Control Technology, Danfoss A/S 1996.

This study is a part of the research project No. T07C 05220 supported by the State Committee for Scientific Research in years 2001-2002 